\title{
A Machine Learning Approach to Beaconing for Vehicular Ad Hoc Network (A Review)
}

\author{
${ }^{1}$ Ramanjeet Kaur Kansla, ${ }^{2}$ Khushboo Bansal \\ ${ }^{1,2}$ CSE Department, Desh Bhagat University, India
}

\begin{abstract}
Vehicular ad hoc network (VANET) are classified as an application of mobile ad hoc network (MANET) that has the potential in improving road safety and in providing traveller comfort.VANET is an emerging field of technology that allows vehicles to communicate together in the absence of fixed infrastructure. The basic premise of VANET is that in order for a vehicle detect other vehicles in the neighborhood.This cognizance, awareness of other vehicles, can be achieved through beaconing. In the near future, many VANET applications will rely on beaconing to enhance information sharing. With respect to change in traffic we have to create an adaptive beaconing rate control mechanism to enable a compromise between network load and precise awareness between vehicles. In this paper we learn to improve the efficiency and accuracy by machine learning approach to beacons.
\end{abstract}

Keywords: VANET, MANET, Beacons, Machine Learning.

\section{Introduction}

In this technical world, every day we get to know about something new in field of technology, new innovation and ideas which revolve around in our daily routine. From telephone to Nano technology everything that discover in this world that are co-related to our mutual need which makes our life easier. In this era, there is vast improvement in the field of transport. There is one technology that plays a vital role in near future that technology is Vehicular ad hoc Network (VANET). It is a kind of wireless ad hoc network, which have high node mobility and fast topology changes. It provides inter-communication between vehicles in absence of fixed infrastructure. VANET provides lot of facilities from safety to navigation and information to entertainment. Due to rapid increase in number of vehicles on roads that resulting in giving birth to unsafe driving. This demands the needs to improve traffic safety and efficiency. For fulfilling this demand, Intelligent Transportation Systems (ITS) have been considered to enable diverse traffic applications such as traffic safety, cooperative traffic monitoring and control of traffic flow. These traffic applications can only be feasible through emerging VANET because vehicular network is recommended as a network environment of ITS. In addition, in the near future more vehicles will be embedded with wireless communication devices such as Wireless Access in Vehicular Environment (WAVE) [1]. In this when a node i.e. vehicle embedded with WAVE, they can communicate by the help of beacons. A particular vehicle exchange beacons messages time by time in this it shares its mobility characteristics with neighbor which leads to creating a cooperative awareness. However, rapid changes in traffic density from sparse to heavy, as well as periodic beaconing between vehicles, can cause the wireless channel between vehicles to promptly become congested, resulting in a high degree of performance degradation of vehicular network [2].The reason for this channel congestion is that each vehicle periodically broadcasts beacons at a fixed rate. This also leads to high channel overloading and hence packet loss. In short, the higher the frequency of beacon rate, the higher the bandwidth overload in dense traffic conditions [3]. This can be resolve by reducing the frequency of beacon generation but after research it conclude that by reducing beacon generation leads to inaccuracy of exchanged position coordinates between nodes, which further result in negative effect on performance of routing protocols and the information that transmitted over beacon of no use because of outdated data. It needs to consider a conditional up-date approach in which a vehicle adapts its beacon rate when there is considerable variation in its neighbor vehicles mobility characteristics. Which have been utilized to design an intelligent ABR approach to control beaconing rate. This is because a fixed beacon rate cannot tackle both bandwidth consumption and accuracy of vehicle status due to rapid changes in vehicular traffic conditions. Therefore, an intelligent ABR approach in V-2-V communication has been developed to tune the beaconing rate in response to changing vehicular traffic characteristics.

ITS applications require awareness with certain qualities. Aside from the contents of the beacon messages (extensively covered in [1]), these qualities are in the spatial and temporal domain, as illustrated in Fig. 1(a). They are:

Range of Awareness - distance up to which a vehicle has information about other vehicles. 
Accuracy of Awareness- freshness (delay) and update rate.

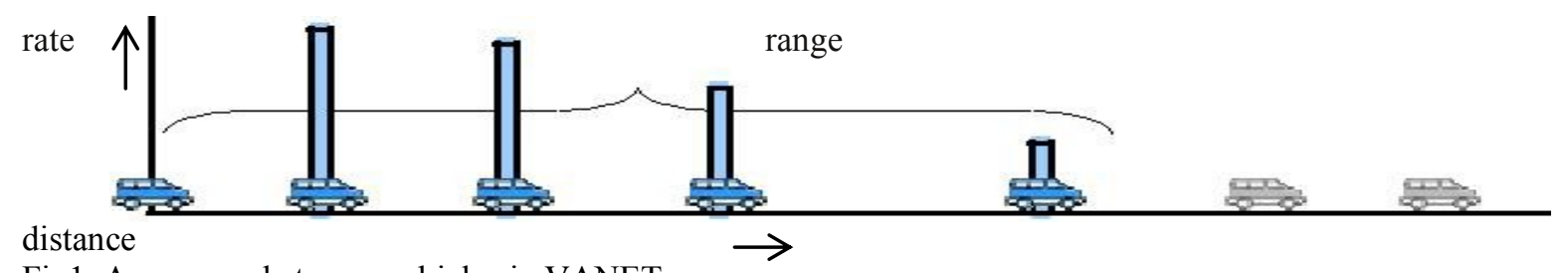

Fig1. Awareness between vehicles in VANET

\section{Related Work}

At present various researches are conducting on the concept of VANET, but these concepts results in showing drawbacks. Beacon control rate (BCR) is the main problem arises from this. This issue can only be resolve by adaptive beaconing rate approach. In this approach beacon generation rate is dependent upon certain things like traffic, lanes, emergency, direction of vehicle etc. The ABR approach is used to synchronize frequency of beacon generation with traffic context. In addition to this various logic are being used like fuzzy logic and machine language, various simulation are implemented and data is to be taken from various researches. Beacon generation rate is more in dense traffic which resulting in loss of packet data and also overloading the whole system but by adopting the ABR approach beacon rate is reduced by implementing some rules. In this rate of generation is reduced by doing this meant to resolve one issue but giving birth to another issue that is error in position of the vehicle. It can be sort out by last beacon message, enhancing positional accuracy between two sequential beacons. This prediction concept is based on current position when a beacon is triggered and an estimated position. When a vehicle determines a change in its physical position, it triggers the next beacon message. In this way, vehicles independently estimate the duration of the next beacon message. However, rapid topology changes of vehicles and mobility traffic characteristics were not considered.

In [4], van Eenennaam et al. proposed architecture to adapt network and MAC-layer parameters in order to mimic the configuration parameters. This adaptive approach can tune MAC layer configurations and beaconing properties to optimal values in the vehicular scenarios. However, vehicular networks are dynamic, as evidenced by dense rush hours and sparse late night traffic conditions. In designing their model, therefore mentioned authors did not take these factors into consideration.

In [5], Fukui et al. proposed a beacon adaptation scheme which considers the distance travelled by vehicles. Moreover, vehicles independently determine the number of lanes the current road has, and the higher the number of lanes, the lower is the beacon rate. In addition, another beacon adaptation technique is based on packet loss rate. But, changing beacon rates based on multi-lane is unfair because multi lanes do not directly imply higher traffic density. Further, the accuracy of information has not been considered.

In [7], Liu Bojin, Khorashadi Behrooz proposed wireless ad hoc mesh network in which size and geographic area they cover. They focused on how the V Meshes could be used to capture and retain certain transient information on the road within a given region of interest for a certain period of time.

In[8],S. Rezaei,first studied the adaptation of beacon rate in order to compromise between information accuracy and bandwidth consumption. After analysis of the parameters which affect the beacon rate, they proposed a scheme to adapt beacon rate according to the VANET traffic behavior.

In [10], Kayhan Zrar Ghafoor proposedan intelligent Adaptive Beaconing Rate (ABR) approach based on fuzzy logic to control the frequency of beaconing by taking traffic characteristics into consideration. The proposed ABR considers the percentage of vehicles traveling in the same direction, and status of vehicles as inputs of the fuzzy decision making system, in order to tune the beaconing rate according to the vehicular traffic characteristics.

\section{Machine Learning Approach}

Machine learning is a system capable of acquiring and integrating the knowledge automatically. The capability of the systems to learn from experience, training, analytical observation, and other means, results in a system that can continuously self-improve and thereby exhibit efficiency and effectiveness. A machine learning system usually starts with some knowledge and a corresponding knowledge organization so that it can interpret, analysis, and test the knowledge acquired.

Machine learning techniques are based on establishing an explicit or implicit model. A singular characteristic of these schemes is the need for labeled data to train the behavioral model, a procedure that places severe demands on resources. In many cases, the applicability of machine learning principles coincides with that for the statistical techniques, although the former is focused on building a model that improves its performance on the basis of previous results. Hence, machine learning for Vehicular Adhoc Network has the ability to change its execution strategy as it acquires new information. This feature could make it desirable to use such schemes 
for all situations.

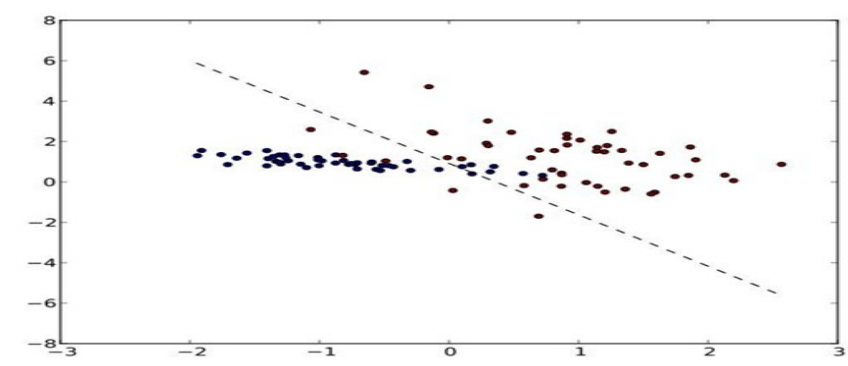

Fig.2: Machine Learning Approach

\section{Proposed Adaptive beaconing approach using Machine Learning approach}

The basic concept of ABR approach is that how a vehicle transmit messages in different situations. In dense traffic it reduces its range of frequency and vice versa. If there is a vehicle that requires an emergency support than the whole system works according to that. These major issues can be resolve by using machine learning approach. By using set of rule and data a machine can adopt to certain situation, easily detection of emergency and non-emergency vehicles this concept can prove to be effective in situation like

\section{A. Status of Vehicle (Emergency or Non-Emergency)}

In real-time environment where there is different type of vehicle having different status are interacting with each other. In these there are some vehicle that have emergency status e.g. police, ambulance etc. these vehicle acquire emergency status which help them to provide special services like vacant lane, information about coming hospital, police station etc. In this machine can respond by using preinstalled set of rules. Figure 1 shows the common vehicular scenario in which the emergency vehicle is enclosed.

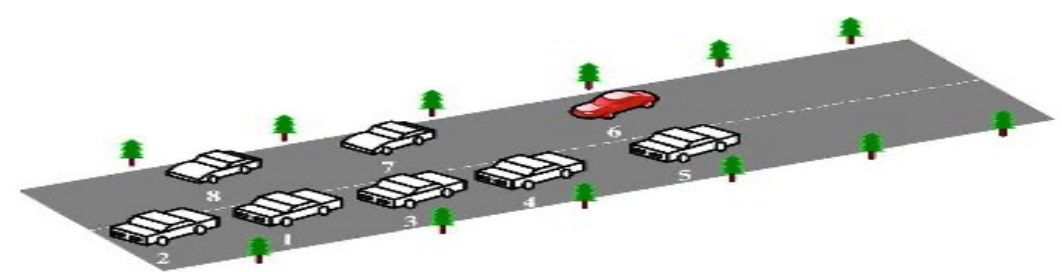

Fig. 3: The emergency (vehicle number 6)/normal (remaining vehicles) Vehicles status and percentage of the same directional Vehicles.

\section{B. Percentage of Directional vehicles}

Rate of beacon depends upon vehicles current status and traffic conditions. In addition to this there are some other attribute that are come under ABR requirements like direction of vehicle, speed, traffic density etc. First, vehicles on the road travel in constrained directions, thus vehicle beacon rate adaptation should take both directions into consideration. For instance, in a vehicular scenario with two ways traffic, and vehicles moving in one direction have congested traffic conditions, they should reduce beacon rate, whereas vehicles moving in the other direction may vary their own beacon rate. Second, the velocity of vehicles and traffic density are implicitly interrelated to one another. This relationship is clearly known in traffic flow theory as in [7] Kerner states that the vehicles average velocity decreases as a result of increasing vehicular traffic density. Therefore, the percentage of vehicles travelling in the same direction is considered as an input as this parameter implicitly combines direction

\section{References}

[1] Wireless access for vehicular environment (wave), December 2010.http://www.standards.its.dot.gov/fact sheet.asp f=80

[2] M. Torrent- Moreno, P. Santi and H. Hartenstein. Distributed fairtransmit power adjustment for vehicular ad hoc networks. In $3^{\text {rd }}$ Annual IEEE Communications Society on Sensor and Ad HocCommunications and Networks, volume 2, pages 479_488. IEEE, 2007

[3] Nzouonta, N. Rajgure, G. Wang, and C. Borcea.Vanet routing on city roads using real-time vehicular traffic information. IEEE Transactions on Vehicular Technology, 58(7):3609-3626, 2009

[4] E. M. van Eenennaam, G. Karagiannis, and G. Heijenk.Towards scalable beaconing in Vanet. In FOURTH ERCIM WORKSHOP ON EMOBILITY, pages 103-108, 2010.

[5] R. Fukui, H. Koike, and H. Okada. Dynamic integrated transmission control(DITRAC) over inter-vehicle communications in ITS. In IEEE Vehicular Technology Conference, volume 1, pages 483-487, 2002

[6] E. M. van Eenennaam, W.K. Wolterink, G. Karagiannis, and G. Heijenk. "Exploring the solution space of beaconing in vanet". In Vehicular Networking Conference (VNC), pages 1-8. IEEE, 2009 
[7] B.S. Kerner. The physics of traffic: empirical freeway patternfeatures, engineering applications, and theory. Springer Verlag, 2004

[8] S. Rezaei, R. Sengupta,H. Krishnan, X. Guan, and P. Student. Adaptive communication scheme for cooperative active safety system, 2008

[9] S. Rezaei, R. Sengupta, H. Krishnan, and X. Guan. Reducingthe communication required by DSRCbased vehicle safety systems. InIEEE Intelligent Transportation Systems Conference (ITSC), pages 361-366, 2007

[10] M.Artimy Local density estimation and dynamic transmission range assignment in vehicular ad hoc networks. IEEE Transactions on Intelligent Transportation Systems, 8(3):400-412, 2007

[11] L. Yang, J. Guo Y. Wu. Channel adaptive one hop broadcasting for VANET. In 11th International IEEE Conference on Intelligent Transportation Systems, pages 369-374, 2008

[12] R. K .Schmidt, T. Leinmuller, E. Schoch, F. Kargl, and G. Schafer. Exploration of adaptive beaconing for efficient intervehicle safety communication.IEEE Network, 24(1):14-19, 2010

[13] B.S. Kerner. The physics of traffic: empirical freeway patternfeatures, engineering applications, and theory. Springer Verlag, 2004

[14] Liu Bojin, Khorashadi Behroojanalysis of the information storage capabilities of Vanet for highway and city traffic in Transportation and Research part C 23 (2012) 68-84.

[15] Schmidt, R. K., Leinmuller., T., Schoch, E., Kargl, F., \& Schafer, G. (2010). Exploration of adaptive beaconing for efficient intervehicle safety communication.IEEE Network, 24(1), 14-19.

[16] S.Marti, T.J. Guili, K.Lai, and M.Baker, Mitigating Routing Misbehaviour in Mobile Ad Hoc Network, Proc. of Mobicom, Boston, August2000.

[17] Kayhan Zrar Ghafoor., Kamalrulnizam Abu Bakar ,Martijn van Eenennaam. Rashid HafeezKhokhar. Alberto J. Gonzalez in Springer science Business Media, LLC 2011 Telecommunication System (2013) 52:139-149

[18] Artimy M, Phillips W, Robertson W. Connectivity with static transmission range in vehicular ad hoc networks. In Proceedings of the 3rd annual communication networks and services research conference. IEEE; 2005, p. 237-42. 